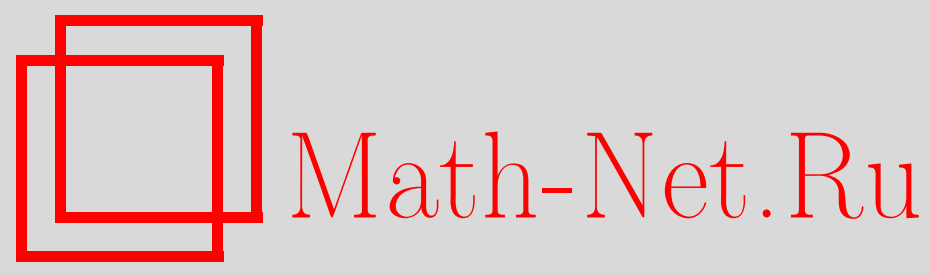

В. В. Корниенко, О спектре вырождающихся операторных уравнений, Матем. заметки, 2000, том 68, выпуск 5, 677691

DOI: https://doi.org/10.4213/mzm989

Использование Общероссийского математического портала Math-Net.Ru подразумевает, что вы прочитали и согласны с пользовательским соглашением http://www . mathnet.ru/rus/agreement

Параметры загрузки:

IP : 54.198 .64 .247

26 апреля 2023 г., 18:00:04

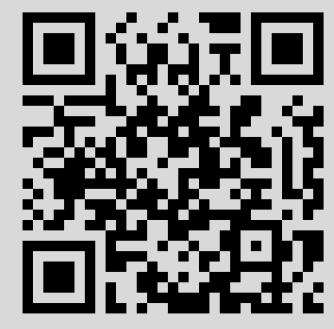




\section{О СПЕКТРЕ ВЫРОЖДАЮЩИХСЯ ОПЕРАТОРНЫХ УРАВНЕНИЙ}

\section{В. В. Корниенко}

Изучено распределение на комплексной плоскости $\mathbb{C}$ спектра оператора $L=L(\alpha$, $a, A), \alpha \in \mathbb{R}, a \in \mathbb{C}$, порожденного замыканием в $H=\mathscr{L}_{2}(0, b) \otimes \mathfrak{H}$ операции $t^{\alpha} a D_{t}^{2}+A$, первоначально заданной на гладких функциях $u(t):[0, b] \rightarrow \mathfrak{H}$ со значениями в гильбертовом пространстве $\mathfrak{H}$, удовлетворяющих условиям Дирихле: $u(0)=u(b)=0$. Здесь $D_{t} \equiv d / d t ; A$ - модельный оператор, действующий в $\mathfrak{H}$. Приведены условия (критерии) на параметр $\alpha$, при выполнении которых собственные функции оператора $L: H \rightarrow H$ образуют полную и минимальную систему, а также базис Рисса в гильбертовом пространстве $H$.

Библиограффия: 14 названий.

Разработанная в [1] концепця обобщенного решения граничной задачи позволила рассматривать левую часть уравнения как некий оператор $L$, действующий в соответствующим образом подобранном функциональном пространстве. В предлагаемой работе язык спектральной теории линейных операторов используется при описании свойств разрешимости модельных граничных задач для некоторого класса уравнений в частных производных, вырождающихся на границе области. Говоря о спектре $\sigma L$ оператора $L$, мы будем придерживаться терминалогии, принятой в [2], [3]. Через $\rho L, P \sigma L, C \sigma L, R \sigma L$ обозначим соответственно резольвентное множество, точечньй, непрерьвньй, остаточный спектры оператора $L$.

\section{1. Постановка задачи}

Изучаемым в данной статье является операторное уравнение

$$
L u \stackrel{\text { def }}{=} a t^{\alpha} D_{t}^{k} u+A u=\lambda u+f,
$$

где $a \in \mathbb{C} ; t \in \overline{V_{t}}, V_{t} \equiv(0, b), 0<b<+\infty ; \alpha \in \mathbb{R} ; D_{t} \equiv d / d t ; k=2$; оператор $A$, коммутирующий с $D_{t}$, действует в некотором комплексном гильбертовом пространстве $\mathfrak{H}$ и удовлетворяет определенным требованиям, формулируемым в терминах спектральной теории операторов (см. начало п. 3.1); $\lambda \in \mathbb{C}, \lambda$ - спектральный параметр. Оператор $L$ порожден замыканием операции $L\left(D_{t}, A\right)=a t^{\alpha} D_{t}^{2}+A$ на гладких функциях $u: \mathbb{R} \rightarrow \mathfrak{H}$, удовлетворяющих условиям Дирихле

$$
\Gamma_{t} u \stackrel{\text { def }}{=} u(0)=u(b)=0
$$

и принадлежащих при всех $t \in \bar{V}_{t}$ области определения оператора $A$. 
Отметим, что регулярньй случай $(\alpha=0)$ достаточно полно исследован в [3]. Там же была сформулирована задача, рассматриваемая в настоящей работе. Уравнение (1), являющееся как сильно $(\alpha>0)$, так и слабо $(\alpha<0)$ иррегулярным [4], дает простейшую ситуацию вырождения типа уравнения. Примером вырождающегося дифференциального уравнения в частных производных, попадающего в поле наших исследований, может служить уравнение

$$
t^{\alpha} \frac{\partial^{2} u(t, x)}{\partial t^{2}}+\Delta_{x} u(t, x)=\lambda u(t, x)+f(t, x),
$$

где $\Delta_{x}$ - самосопряженньй полуограниченный эллиптический оператор, порожденньй регулярной краевой задачей в ограниченной области $\Omega$ с достаточно гладкой границей; $(t, x) \in \bar{V}, V=V_{t} \times \Omega$.

Используемьй нами метод изучения уравнения (1) позволяет рассмотреть вначале одномерный случай, т.е. случай, в котором $A$ - умножение на константу $A(s) \in \mathbb{C}$, где $s-$ "номер", приписьваемьй “проекции" уравнения (1) в соответствующее подпространство (см. (20а)). Техника перехода к дифференциально-операторному уравнению, развитая в [3] и использованная, например, в [5] при изучении соответствующих вопросов для уравнений смешанного типа, позволяет рассмотреть в дальнейшем общее уравнение (1), т.е. уравнение, в котором $A$ является $M$-оператором [3, с. 100]. Спектральные характеристики уравнения (1) при $k=1$ были исследованы в [6]. Характер граничных условий по $t$ (при $t=0, b)$, присоединяемых к вырождающемуся дифференциально-операторному уравнению второго порядка по $t:-D_{t} t^{\alpha} D_{t} u-D_{t} A u-P u=f$ и обеспечивающих существование и единственность решения при любых правых частях, изучался в [7]. В свете проводимых там исследований уравнение (1) - несамосопряженное уравнение второго типа (при $\alpha=1$ ). Вырождаюшиеся дифференциально-операторные уравнения более высокого порядка по $t$ исследовались, например, в [8]. Перейдем непосредственно к изложению результатов.

\section{2. Одномерный случай}

2.1. Структура фундаментальной системы решений. Рассмотрим обыкновенное дифференциальное уравнение

$$
t^{\alpha} D_{t}^{2} v-\varrho v=0, \quad \varrho \in \mathbb{C} .
$$

Исследуем поведение функций $v_{1}(t, \varrho), v_{2}(t, \varrho)$, образующих $\Phi$ СР (фундаментальную систему решений) уравнения (3) в окрестности $t=0$. Определим функцию $F: \mathbb{R}^{2} \rightarrow \mathbb{R}$, $F(n, \alpha)=n(2-\alpha)[(n(2-\alpha)+1]$, областью определения $\mathfrak{D}(F)$ которой является набор пар $(n, \alpha)$ чисел $n= \pm 1, \pm 2, \ldots ; \alpha \in \mathbb{R}$, т.е. $\mathfrak{D}(F)=\{(n, \alpha): n \in \mathbb{Z} \backslash\{0\}, \alpha \in \mathbb{R}\}$. Отметим, что $(F(n, \alpha)=0) \Longleftrightarrow(\alpha=2) \vee(\alpha=2+1 / \nu) \wedge(n=\nu)$, где $\nu= \pm 1, \pm 2, \ldots$

ЛЕмма 1. ФСР $\left\{v_{k}(t, \varrho) ; k=1,2\right\}$ уравнения (3) может быть представлена в виде

$$
v_{1}(t, \varrho)= \begin{cases}t^{1 / 2+\sqrt{1 / 4+\varrho},} & \text { если } \alpha=2, \\ t \sum_{n=0}^{\infty} \alpha_{n} t^{n(2-\alpha)}, & \text { если }(\alpha \neq 2) \wedge\left(\alpha \neq 2+\frac{1}{\nu}\right) \\ \sum_{n=0}^{\infty}\left(\gamma_{n} t+\beta_{n} \ln t\right) t^{n(2-\alpha)}, & \text { если } \alpha=2+\frac{1}{\nu}\end{cases}
$$


где $\nu=1,2,3, \ldots ; \alpha_{0}, \gamma_{0}, \gamma_{\nu}$ - произвольные не равные нулю числа, а остальные коэффичиенты определяются из следующих соотношений:

$$
\begin{aligned}
& \alpha_{n}=\frac{\varrho}{F(n, \alpha)} \alpha_{n-1} \quad \text { npu } n=1,2,3, \ldots, \\
& \text { если } \nu>1, \text { mo } \gamma_{n}=\frac{\varrho}{F(n, \alpha)} \gamma_{n-1} \text { nрu } n=1, \ldots, \nu-1 \text {, } \\
& \gamma_{\nu+n}=\frac{\varrho \gamma_{\nu+n-1}-[2 n(2-\alpha)-1] \beta_{n}}{F(\nu+n, \alpha)} \quad \text { npu } n=1,2,3, \ldots \text {, } \\
& \beta_{0}=-\varrho \gamma_{\nu-1}, \quad \beta_{n}=\frac{\varrho}{F(-n, \alpha)} \beta_{n-1} \quad \text { npu } n=1,2,3, \ldots \text {; } \\
& v_{2}(t, \varrho)= \begin{cases}t^{1 / 2-\sqrt{1 / 4+\varrho},} & \text { если }(\alpha=2) \wedge\left(\varrho \neq-\frac{1}{4}\right), \\
t^{1 / 2} \ln t, & \text { если }(\alpha=2) \wedge\left(\varrho=-\frac{1}{4}\right), \\
\sum_{n=0}^{\infty} \beta_{n} t^{n(2-\alpha)}, & \text { если }(\alpha \neq 2) \wedge\left(\alpha \neq 2-\frac{1}{\nu}\right), \\
\sum_{n=0}^{\infty}\left(\delta_{n}+\alpha_{n} t \ln t\right) t^{n(2-\alpha)}, & \text { если } \alpha=2-\frac{1}{\nu},\end{cases}
\end{aligned}
$$

где $\nu=1,2,3, \ldots ; \beta_{0}, \delta_{0}, \delta_{\nu}$ - произвольные не равные нулю числа, а остальные коэффичиенты определяются из следующих соотношений:

$$
\begin{gathered}
\beta_{n}=\frac{\varrho}{F(-n, \alpha)} \beta_{n-1} \quad \text { npu } n=1,2,3, \ldots, \\
\text { если } \nu>1, \text { mо } \delta_{n}=\frac{\varrho}{F(-n, \alpha)} \delta_{n-1} \\
\delta_{\nu+n}=\frac{\varrho \delta_{\nu+n-1}-[2 n(2-\alpha)+1] \alpha_{n}}{F(-(\nu+n), \alpha)} \quad \text { npu } n=1, \ldots, \nu-1,2,3, \ldots, \\
\alpha_{0}=-\varrho \delta_{\nu-1}, \quad \alpha_{n}=\frac{\varrho}{F(n, \alpha)} \alpha_{n-1} \quad \text { nри } n=1,2,3, \ldots .
\end{gathered}
$$

ДоКАЗАТЕЛЬСТво. Если $\alpha=2$, то мы имеем дело с уравнением Эйлера, торого находится известньми методами [9, с. 228]. В случае $\alpha \neq 2$ ФСР уравнения (3) будем искать методом, используемым при нахождении решений уравнений класса $\Phi$ укса с регулярной особой точкой $[9$, с. 236$]$. Именно, решения уравнения (3) ищем в форме рядов

$$
v_{1}(t, \varrho)=t \sum_{n=0}^{\infty} \alpha_{n} t^{n \varkappa}, \quad v_{2}(t, \varrho)=\sum_{n=0}^{\infty} \beta_{n} t^{n \varkappa},
$$

при этом уравнение для определения показателя $\varkappa$ (уравнение с неизвестной $\varkappa$ возникает при подстановке рядов (4) в уравнение (3)) имеет вид $n \varkappa-1+\alpha=(n-1) \varkappa+1$, из которого находим $\varkappa=2-\alpha$.

Пусть $\alpha \neq 2+1 / \nu$, где $\nu= \pm 1, \pm 2, \pm 3, \ldots$ Подставляя ряды (4) в уравнение (3), собирая подобные члены и приравнивая нулю коэффициенты при различных степенях $t$, получим последовательности рекуррентных соотношений для определения чисел $\alpha_{n}$ 
и $\beta_{n}$. Если $\alpha=2+1 / \nu$, где $\nu= \pm 1, \pm 2, \pm 3, \ldots$, то определяющее уравнение $F(n, \alpha)=0$ обращается в нуль при $n=\nu$. При $\nu=1,2,3, \ldots(\nu=-1,-2,-3, \ldots)$ функция $v_{2}(t, \varrho)$ $\left(v_{1}(t, \varrho)\right)$, входящая в состав $Ф$ СР уравнения $(3)$, останется неизменной, а функцию $v_{1}(t, \varrho)\left(v_{2}(t, \varrho)\right)$ будем искать в виде ряда $(4)$ с “поправочным" (с точностью до некоторой постоянной $C$ ) слагаемым - произведения $\ln t$ и первой найденной функции, т.е. в виде

$$
v_{m}(t, \varrho)=\sum_{n=0}^{\infty}\left((2-m) \gamma_{n}+(m-1) \delta_{n}\right) t^{n(2-\alpha)+1}+C \cdot \ln t \cdot v_{3-m}(t, \varrho), \quad m=1,2
$$

При этом, как и ранее, последовательности рекуррентных соотношений для определения чисел $\gamma_{n}$ (при $\nu=1,2,3, \ldots$ ) или $\delta_{n}$ (при $\nu=-1,-2,-3, \ldots$ ) определим, пользуясь уравнением (3). (Отметим, что рекуррентные соотношения для определения чисел $\alpha_{n}$ (при $\alpha=2+1 / \nu$, где $\nu=-1,-2,-3, \ldots$ ) и $\beta_{n}($ при $\alpha=2+1 / \nu$, где $\nu=1,2,3, \ldots)$, а следовательно, для $\delta_{n}$ и $\gamma_{n}$ соответственно вьписаны нами несколько в ином ("равносильном") виде.)

2.2. Свойства решений операторного уравнения $L_{s} v=g$. Изучим в гильбертовом пространстве $H_{t} \equiv H\left(V_{t}\right)$ комплекснозначных функций $v: \mathbb{R} \rightarrow \mathbb{C}$ с интегрируемым квадратом на $V_{t}$ уравнение

$$
L_{s} v \stackrel{\text { def }}{=} a t^{\alpha} D_{t}^{2} v+A(s) v=\lambda v+g, \quad v, g \in H_{t} .
$$

Пусть $\mathfrak{D}_{t}$ - линейное многообразие гладких функций, удовлетворяющих условиям (2), а точнее

$$
\mathfrak{D}_{t} \stackrel{\text { def }}{=}\left\{v:\left(v \in C\left(\bar{V}_{t}\right) \cap C^{2}\left(V_{t}\right)\right) \wedge\left(\Gamma_{t} v=0\right)\right\} .
$$

ОПРЕДЕЛЕНИЕ 1. Элемент $v \in H_{t}$ называется обобщенным решением уравнения $(5)$, если существует последовательность $\left\{v_{n}\right\} \stackrel{\text { def }}{=}\left\{v_{n} ; n \in \mathbb{N}\right\}$ функций $v_{n}=v_{n}(t)$ из $\mathfrak{D}_{t}$ такая, что вьполнены равенства

$$
\lim _{n \rightarrow \infty}\left|v_{n}-v ; H_{t}\right|=\lim _{n \rightarrow \infty}\left|L_{s}\left(D_{t}\right) v_{n}-\lambda v-g ; H_{t}\right|=0
$$

Определение 1 решения уравнения (5) породило замкнутьй оператор $L_{s}: H_{t} \rightarrow H_{t}$. Именно, $v \in \mathfrak{D}\left(L_{s}\right)$ и $L_{s} v=\lambda v+g$, если вьполнены условия определения 1 . Изучим функциональную зависимость спектра и свойств системы собственных функций (коротко СС $\Phi)$ оператора $L_{s}=L_{s}(\alpha, a)$ от параметров $\alpha, a$. Приведем вначале легко доказываемые свойства элементов области определения $\mathfrak{D}\left(L_{s}\right)$ оператора $L_{s}$.

Лемма 2. Пусть в - произвольное положительное число из $V_{t}$. Любой әлемент $v$ из области определения $\mathfrak{D}\left(L_{s}\right)$ оператора $L_{s}=L_{s}(\alpha, a), \alpha \in \mathbb{R}$, представим функцией $v(t)$, первая производная $D_{t} v(t)$ которой абсолютно непрерывна на $\bar{V}_{t}^{\varepsilon}$, а сама функиия $v(t)$ удовлетворяет условию $v(b)=0$. При $\alpha \leqslant 3 / 2 \oint y н к-~$ иия $v(t)$ непрерывна на $\bar{V}_{t}$ и удовлетворяет условиям Дирихле: $v(0)=v(b)=0$. При $\alpha<1 / 2$ первая производная $D_{t} v(t)$ функиии $v(t)$ абсолютно непрерывна на $\bar{V}_{t}$. 
Лемма 3. Собственная функиия $v_{s, \lambda}(t)$ оператора $L_{s}$, соответствующая собственному значению $\lambda$, может быть представлена в виде

$$
v_{s, \lambda}(t)=\Delta(t, b, \varrho),
$$

әде

$$
\Delta(t, \tau, \varrho)=\left|\begin{array}{ll}
v_{1}(t, \varrho) & v_{2}(t, \varrho) \\
v_{1}(\tau, \varrho) & v_{2}(\tau, \varrho)
\end{array}\right|, \quad 0<\tau, t \leqslant b, \quad \varrho=\frac{\lambda-A(s)}{a} .
$$

При $\alpha \leqslant 3 / 2$ собственная функиия $v_{s, \lambda}(t) \in \mathfrak{D}_{t}$.

Из лемм 2,3 следует, что при $\alpha<2$ решения уравнения

$$
\widetilde{\Delta}_{s}(\lambda) \stackrel{\text { def }}{=} \widehat{\Delta}(\varrho) \stackrel{\text { def }}{=} \Delta(0, b, \varrho)=0
$$

безусловно являются собственными значениями оператора $L_{s}$. Решения уравнения (7), естественно, будем искать асимптотическими методами. С этой целью получим интегральные представления $\Phi$ СР уравнения (3). Именно, используем функцию $\mathfrak{w}(z)=$ $(z-i)^{\alpha /(2-\alpha)}(z+i)^{\alpha /(2-\alpha)}$ однозначную и регулярную на плоскости $\mathbb{C}$ с разрезами $l_{k}=\left\{z: z=t+i(-1)^{k+1},-\infty<t \leqslant 0\right\}, k=1,2$, т.е. на множестве $\mathbb{C} \backslash l_{1} \backslash l_{2}$, для представления $\Phi C P$ уравнения (3) в виде контурных интегралов. При этом мы будем считать ее продолженной по непрерьвности на верхний и нижний берега каждого из разрезов $l_{k}$, т.е. считаем функцию $w: \mathbb{C} \rightarrow \mathbb{C}$ непрерьвной в области $\mathbb{C} \backslash l_{1} \backslash l_{2}$ вплоть до границы. (В работе (по определению) считаем, что $z^{v}=e^{v \ln z}, \ln z=\ln |z|+i \arg z$, $-\pi<\arg z \leqslant \pi$.) Обозначим через $l_{k}^{\prime}$ кривую, состоящую из кусков верхнего, нижнего берегов разреза $l_{k}$ и окружности радиуса $\varepsilon>0$ с центром в точке $i(-1)^{k+1}$, т.е.

$$
\begin{aligned}
& l_{k}^{\prime}=\left\{z_{k}(\tau) ;-\infty<\tau<+\infty\right\}, \\
& z_{k}(\tau)=i(-1)^{k+1}+ \begin{cases}-\frac{\varepsilon}{\pi} \tau, & \text { если } \pi<\tau<+\infty, \\
\varepsilon(\cos \tau+i \sin \tau), & \text { если }-\pi \leqslant \tau \leqslant+\pi, \\
\frac{\varepsilon}{\pi} \tau, & \text { если }-\infty<\tau<-\pi,\end{cases}
\end{aligned}
$$

где $\varepsilon \ll 1 / 2$, а “《” -знак достаточной малости. При этом мы считаем, что движение по кривой (8), соответствующее возрастанию параметра $\tau$, начинается на нижнем и заканчивается на верхнем берегах разреза $l_{k}$.

Лемма 4. Пусть $\alpha<2$. ФСР $\left\{\mathfrak{v}_{k}(t, \varrho) ; k=1,2\right\}$ уравнения (3) может быть представлена в виде

$$
\mathfrak{v}_{k}(t, \varrho)=\gamma^{\nu} t \int_{l_{k}^{\prime}} \mathfrak{w}(z) e^{\gamma t^{\beta} z} d z
$$

əде $\gamma=2 \nu \sqrt{-\varrho}, \nu=1 /(2-\alpha), \beta=(2-\alpha) / 2$. 
ДокАЗАТЕЛьСтво. Достаточно заметить, что уравнение (3) заменой $v=\gamma^{\nu} t \cdot w$, $z=\gamma t^{\beta}$ сводится к уравнению

$$
z D_{z}^{2} w+(2 \nu+1) D_{z} w+z w=0
$$

ФСР которого может быть найдена преобразованием Лапласа

$$
w_{k}(z)=\int_{l_{k}^{\prime}} \mathfrak{w}(t) e^{z t} d t
$$

Отметим, что представление (10) решения уравнения (9) справедливо $\forall z \in \mathbb{C}(-\pi<$ $\arg z<\pi)$. Это соответствует всем значениям спектрального параметра $\varrho \in \mathbb{C}$ уравнения (3), кроме неотрицательных значений, т.е. $\forall \varrho\left((\varrho \in \mathbb{C}) \wedge\left(\varrho \notin \mathbb{R}_{+} \cup\{0\}\right)\right)$. Для получения аналитического продолжения полученного решения (10) уравнения (9) в более широкую область значений переменного $z \in \mathbb{C}$ (позволяющее охватить положительные значения $\varrho$, т.е. значения $\varrho \in \mathbb{R}_{+}$) достаточно повернуть разрез $l_{k}$ (а вместе с ним и контур $\left.l_{k}^{\prime}\right)$, по часовой стрелке вокруг точки $z_{k}=i(-1)^{k+1}$ на некоторьй достаточно малый угол $\alpha(0<\alpha<\pi / 2)$.

2.3. Спектр оператора $L_{s}$. Прежде чем вьписать асимптотическую формулу распределения собственных значений оператора $L_{s}$, являюшихся решением уравнения $(7)$, приведем качественную картину расположения нулей функции $\widehat{\Delta}(\varrho)$ на $\mathbb{C}$.

ПРЕДЛОЖЕНИЕ 1. Если $\alpha<2 u \widehat{\Delta}(\varrho)=0, m o \operatorname{Re} \varrho<0 u \operatorname{Im} \varrho=0$.

ДокАЗАТЕЛЬство. Пусть собственному значению $\lambda \in \mathbb{C}, \widetilde{\Delta}_{s}(\lambda)=0$, оператора $L_{s}$ соответствует собственная функция $v=v(t, \varrho), \varrho=(\lambda-A(s)) / a$. Умножим обе части равенства $L_{s} v=\lambda v$ на $\bar{v}$ и проинтегрируем по промежутку $V_{t}$. Учитьвая свойства $\Phi С Р$ уравнения (5) в окрестности $t=0$, получим

$$
\varrho \int_{0}^{b} \frac{|v|^{2}}{t^{\alpha}} d t=-\int_{0}^{b}\left|D_{t} v\right|^{2} d t<0
$$

т.е. $\operatorname{Re} \varrho<0, \operatorname{Im} \varrho=0$.

Следующие предложения дают как количественную, так и качественную картину расположения спектра оператора $L_{s}$ на $\mathbb{C}$.

ПРЕДЛОЖЕНИЕ 2. Если $\alpha<2$, то множсество $\left\{\lambda_{k, s}: k=1,2,3, \ldots\right\}$ собственных значений $\lambda_{k, s}$ оператора $L_{s}$, являюшихся решением уравнения (7), не имеет конечной предельной точки и допускает асимптотическое $($ при $k \rightarrow \infty)$ nредставление вида

$$
\lambda_{k, s}=-a\left(\frac{(2-\alpha)^{2} \pi^{2}}{4 b^{2-\alpha}} k^{2}+\frac{\alpha(2-\alpha) \pi^{2}}{8 b^{2-\alpha}} k+\frac{\alpha(4-\alpha)}{16 b^{2-\alpha}}+\frac{\alpha^{2} \pi^{2}}{64 b^{2-\alpha}}\right)+A(s)+O\left(\frac{1}{k}\right) .
$$


ДокАЗАТЕЛЬСТВо. Воспользовавшись методом асимптотических разложений решений, полученных преобразованием Лапласа [10, с. 408], получим асимптотическое разложение функции $v_{1}(b, \varrho)$ при $\varrho \rightarrow-\infty$ и уравнение

$$
\cos \left(\gamma b^{\beta}-\frac{\pi}{2} \nu-\frac{\pi}{4}\right)-\frac{p_{1} \cdot(\nu+1 / 2)}{2 \gamma b^{\beta}} \sin \left(\gamma b^{\beta}-\frac{\pi}{2} \nu-\frac{\pi}{4}\right)+O\left(\frac{1}{\varrho}\right)=0
$$

равносильное уравненнию (7). Решим его.

Рассмотрим ( "невозмущенное") уравнение

$$
\cos \left(z-z_{0}\right)-\frac{\mathfrak{a}_{0}}{z} \sin \left(z-z_{0}\right)=0
$$

в котором

$$
z_{0}=\frac{\pi}{2} \nu+\frac{\pi}{4}, \quad \mathfrak{a}_{0}=\frac{1}{2} p_{1} \cdot\left(\nu+\frac{1}{2}\right)=\frac{1}{2}\left(\nu^{2}-\frac{1}{4}\right), \quad z>0 .
$$

Решения $z=z_{k}^{\prime}, k=-N,-N+1,-N+2, \ldots$, уравнения (11) действительны и могут быть представлены в виде

$$
z_{k}^{\prime}=z_{0}-\frac{\pi}{2}+k \pi+\varsigma_{k}
$$

где $\left|\varsigma_{k}\right|<\pi / 2$, причем $N=N(\alpha) \in \mathbb{N}$ таково, что $z_{-N}^{\prime}>0$. Найдем асимптотику $z_{k}^{\prime}$ при $k \rightarrow \infty$. Полагая в (11) $z=z_{0}-\pi / 2+k \pi+\varsigma ; \varepsilon=\varepsilon(k), \varepsilon(k)=\mathfrak{a}_{0} /(k \pi)$, получим для ऽ уравнение $\mathscr{F}(\varsigma)+\varepsilon=0$, в котором функция

$$
\mathscr{F}(\varsigma)=\frac{2 \mathfrak{a}_{0} \sin \varsigma}{2 \mathfrak{a}_{0} \cos \varsigma+\left(2 z_{0}-\pi+2 \varsigma\right) \sin \varsigma}
$$

регулярна $(\varsigma \in \mathbb{C})$ в точке $\varsigma=0$ и $\mathscr{F}^{\prime}(0)=1$. Следовательно, в силу теоремы об обратной функции существует функция $\varsigma=\varsigma(\varepsilon)$, обратная к функции $\mathscr{F}(\varsigma)$, регулярная в точке $\varepsilon=0$. Подставляя ряд Тейлора $\varsigma(\varepsilon)=\sum_{n=1}^{\infty} c_{n} \varepsilon^{n}$ функции $\varsigma(\varepsilon)$, сходящийся в круге $|\varepsilon|<\varepsilon_{0}$ при достаточно малом $\varepsilon_{0}>0$, в формулу $(12)$, получим представление решений уравнения (11):

$$
z_{k}^{\prime}=z_{0}-\frac{\pi}{2}+k \pi+\frac{\mathfrak{a}_{0}}{k \pi}+\sum_{n=2}^{\infty} c_{n}\left(\frac{\mathfrak{a}_{0}}{k \pi}\right)^{n}
$$

справедливое при достаточно больших $k$.

Рассмотрим теперь исходное уравнение

$$
\cos \left(z-z_{0}\right)-\frac{\mathfrak{a}_{0}}{z} \sin \left(z-z_{0}\right)+O\left(\frac{1}{z^{2}}\right)=0, \quad z=\gamma b^{\beta} .
$$

В силу теоремы Коши (о промежуточном значении) решения $z=z_{k}$ уравнения (13) при достаточно больших $k$ можно записать в виде $z_{k}=z_{k}^{\prime}+z_{k}^{\prime \prime}$, где $\left|z_{k}^{\prime \prime}\right|<\pi / 2$. Найдем асимптотику $z_{k}^{\prime \prime}$ (а, следовательно, и $z_{k}$ ) при $k \rightarrow \infty$. Подставляя в уравнение (13) $z=z_{k}$, сведем его к уравнению относительно $z_{k}^{\prime \prime}$. Именно, используя равенство (11) (при $z=z_{k}^{\prime}$ ), а также формулы

$$
\sin \left(z_{k}^{\prime}-z_{0}\right)=(-1)^{k}\left(1+O\left(\frac{1}{k^{2}}\right)\right), \quad \cos \left(z_{k}^{\prime}-z_{0}\right)=O\left(\frac{1}{k}\right)
$$


после приведения подобных уравнение (13) примет вид

$$
\sin \left(z_{k}^{\prime \prime}\right)=O\left(\frac{1}{k^{2}}\right)
$$

Откуда $z_{k}^{\prime \prime}=O\left(1 / k^{2}\right)$. Следовательно,

$$
z_{k}=z_{0}-\frac{\pi}{2}+k \pi+\frac{\mathfrak{a}_{0}}{k \pi}+O\left(\frac{1}{k^{2}}\right)
$$

Учитьвая, что $\lambda_{k, s}=-a \varrho_{k}+A(s)$, где $\varrho_{k}=(2-\alpha)^{2} z_{k}^{2} /\left(4 b^{2-\alpha}\right)$, получим асимптотическое представление собственных значений оператора $L_{s}$.

Обозначим через $C[0, b]$ подмножество $C[0, b]$, состоящее из функций, финитных в окрестности $t=0$ (равных нулю локально при $t \rightarrow+0$ ), т.е.

$$
C[0 \circ, b] \stackrel{\text { def }}{=}\left\{v:(v \in C[0, b]) \wedge\left(\exists \varepsilon_{v} \in(0, b) \forall t \quad\left(t \in\left(0, \varepsilon_{v}\right) \Longrightarrow v(t)=0\right)\right)\right\}
$$

через

$$
\mathbb{P}_{s}=\left\{\lambda: 4 \operatorname{Re} \frac{\lambda-A(s)}{a}<-\operatorname{Im}^{2} \frac{\lambda-A(s)}{a}+3\right\}
$$

- внутренность параболы.

ПРЕДЛОЖЕНИЕ 3. Если $\alpha=2, \operatorname{moP} \operatorname{Po} L_{s}=\mathbb{P}_{s}$.

ДокАЗАтЕЛьство. Легко видеть, что для оператора $L_{s}$ число $\lambda=a \varrho+A(s)$, где $\varrho \in \mathbb{P}=\left\{\varrho: 4 \operatorname{Re} \varrho<-\operatorname{Im}^{2} \varrho+3\right\}$, есть собственное значение с соответствующей ему собственной функцией

$$
v(t, \varrho)=t^{\frac{1}{2}} \begin{cases}\frac{\operatorname{sh}\{\sqrt{1 / 4+\varrho}(\ln b-\ln t)\}}{\sqrt{1 / 4+\varrho},} & \varrho \neq-\frac{1}{4} \\ \ln b-\ln t, & \varrho=-\frac{1}{4}\end{cases}
$$

Причем если $\varrho \in \mathbb{P}_{0}=\left\{\varrho: \operatorname{Re} \varrho<-\operatorname{Im}^{2} \varrho\right\}$, то (14) является "классической" собственной функцией $[10]$, т.е. $v \in \mathfrak{D}_{t}$. С другой стороны, если $\lambda=a \varrho+A(s)$, где $\varrho \notin \mathbb{P}$, то решение уравнения (5), например, для любой функции $g \in C\left[0{ }^{\circ}, b\right]$ существует и единственно.

Отметим еще ряд спектральных свойств рассматриваемой задачи: $(\alpha=2) \Longrightarrow$ $\left(R \sigma L_{s}=\varnothing\right) ;(\alpha<2) \Longrightarrow\left(C \sigma L_{s}=\varnothing\right) \vee\left(R \sigma L_{s}=\varnothing\right)$. Первое свойство получено в ходе доказательства предложения 3 , второе вытекает из определения и структуры решения уравнения (5). 
2.4. Свойства ССФ оператора $L_{s}$. Займемся изучением свойств системы собственных функций оператора $L_{s}$. Пусть оператор $L_{s}^{T}: H_{t} \rightarrow H_{t}$ порожден (как и оператор $L_{s}$ ) замыканием операции $L_{s}^{T}\left(D_{t}\right)$,

$$
L_{s}^{T}\left(D_{t}\right) \stackrel{\text { def }}{=} \bar{a} t^{\alpha} D_{t}^{2}+2 \bar{a} \alpha t^{\alpha-1} D_{t}+\left(\bar{a} \alpha(\alpha-1) t^{\alpha-2}+\overline{A(s)}\right) \cdot I
$$

первоначально заданной на линейном многообразии $\mathfrak{D}_{t}^{T}$,

$$
\mathfrak{D}_{t}^{T} \stackrel{\text { def }}{=}\left\{v:\left(v \in C^{2}\left(V_{t}\right)\right) \wedge\left(\Gamma_{t}^{T} v=0\right)\right\}
$$

где

$$
\Gamma_{t}^{T} v \stackrel{\text { def }}{=}\left\{\begin{array}{l}
\lim _{t \rightarrow+0} t^{\alpha} v(t), \\
\lim _{t \rightarrow b-0} v(t) .
\end{array}\right.
$$

Отметим, что операция (15) является формально сопряженной [11] с операцией левой части уравнения (5).

Легко доказываются следующие ниже

ЛЕмма 5. Любой әлемент $v$ из области определения $\mathfrak{D}\left(L_{s}^{T}\right)$ оператора $L_{s}^{T}=$ $L_{s}^{T}(\alpha, a), \alpha \in \mathbb{R}$, представим функиией $v(t)$, для которой имеют место вложения $t^{\alpha} v(t) \in C^{1}\left(\bar{V}_{t}\right) \cap C^{2}\left(V_{t}\right)$ и выполнены условия $\Gamma_{t}^{T} v=0$.

ЛЕмма 6. Собственная функиия $v_{s, \bar{\lambda}}(t)$ оператора $L_{s}^{T}=L_{s}^{T}(\alpha, a), \alpha \in \mathbb{R}$, соответствующая собственному значению $\bar{\lambda} \in \mathbb{C}$, может быть представлена в виде

$$
v_{s, \bar{\lambda}}(t)=t^{-\alpha} \Delta(t, b, \bar{\varrho})
$$

ПРЕДЛОЖЕНИЕ 4. Пусть $\alpha<3 / 2$. Если $v(t)-$ собственная функиия оператоpa $L_{s}$, соответствующая его собственному значению $\lambda$, то $t^{-\alpha} \overline{v(t)}-$ собственная функиия оператора $L_{s}^{T}$, соответствующая его собственному значению $\bar{\lambda}$. И наоборот, при $\alpha \geqslant 3 / 2$ имеем равенство $P \sigma L_{s}^{T}=\varnothing$.

ПреДЛОЖЕНИЕ 5. Пусть $\alpha<2$. Совокупность собственныХ функиий оператоpа $L_{s}$ образует:

а) полную систему в $H_{t}$;

б) (минимальную систему в $\left.H_{t}\right) \Longleftrightarrow(\alpha<3 / 2)$;

в) (гильбертову систему в $\left.H_{t}\right) \Longleftrightarrow(0 \leqslant \alpha<3 / 2)$;

г) (бесселеву систему в $\left.H_{t}\right) \Longleftrightarrow(\alpha \leqslant 0)$.

ДоказАТЕльство. Обозначим через $\widetilde{v}_{1}(t), \widetilde{v}_{2}(t)$ нетривиальные решения обыкновенного дифференциального уравнения

$$
t^{\alpha} D_{t}^{2} v-v=0
$$

удовлетворяющие условиям

$$
\widetilde{v}_{1}(0)=\widetilde{v}_{2}(b)=0
$$


Отметим, что в рассматриваемом нами случае (т.е. $\alpha<2$ ) решения уравнения (16), удовлетворяющие условиям (17), существуют

$$
\widetilde{v}_{1}(t)=v_{1}(t, \varrho), \quad \widetilde{v}_{2}(t)=\Delta(t, b, \varrho) \quad \text { при } \varrho=1 .
$$

Предполагая их линейную зависимость, получим равенство

$$
0=\int_{0}^{b}\left(\widetilde{v}_{1} D_{t}^{2} \widetilde{v}_{1}-\frac{\widetilde{v}_{1}^{2}}{t^{\alpha}}\right) d t=-\int_{0}^{b}\left[\left(D_{t} \widetilde{v}_{1}\right)^{2}+\frac{\widetilde{v}_{1}^{2}}{t^{\alpha}}\right] d t .
$$

Откуда $\forall t \in \bar{V}_{t}\left(\widetilde{v}_{1}(t)=\widetilde{v}_{2}(t)=0\right)$.

Положим

$$
G(\tau, t)=\frac{1}{W} \begin{cases}\widetilde{v}_{1}(\tau) \widetilde{v}_{2}(t), & 0 \leqslant \tau \leqslant t \leqslant b, \\ \widetilde{v}_{1}(t) \widetilde{v}_{2}(\tau), & 0 \leqslant t \leqslant \tau \leqslant b,\end{cases}
$$

где $W$ - вронскиан выбранной системы функций $\widetilde{v}_{1}(t), \widetilde{v}_{2}(t)$. Используя свойства $\Phi С Р$ уравнения (3), нетрудно проверить, что уравнение $L_{s} v=\lambda v$, рассматриваемое над $\mathfrak{D}_{t}$, эквивалентно уравнению

$$
v=(\varrho-1) B \widetilde{L} B^{-1} v,
$$

в котором $B$ - оператор умножения на функцию $\sqrt{t^{\alpha}}, \widetilde{L}$-интегральный оператор с ядром $G(\tau, t) /\left(\sqrt{\tau^{\alpha}} \sqrt{t^{\alpha}}\right)$, т.е.

$$
B, \widetilde{L}: H_{t} \rightarrow H_{t}, \quad B v=\sqrt{t^{\alpha}} v, \quad \widetilde{L} w=\int_{0}^{b} \frac{G(\tau, t)}{\sqrt{\tau^{\alpha}} \sqrt{t^{\alpha}}} w(\tau) d \tau .
$$

Рассмотрим теперь уравнение

$$
w=(\varrho-1) \widetilde{L} w
$$

вытекающее из (18), в котором надо положить $w=B^{-1} v$. Ядро оператора $\widetilde{L}$ пусто. Кроме того, оператор $\widetilde{L}$ является отрицательным самосопряженным, вполне непрерывным оператором в гильбертовом пространстве $H_{t}$. Следовательно, совокупность его пронормированных собственных функций $w_{k}(t), k=1,2,3, \ldots$, образует полную ортонормальную систему в $H_{t}$. Учитьвая связь, установленную благодаря уравнению (18) между операторами $L_{s}$ и $\widetilde{L}$, выясним теперь свойства системы

$$
v_{k}=B w_{k}, \quad k=1,2,3, \ldots,
$$

собственных функции оператора $L_{s}$, соответствующих его собственным значениям $\lambda_{k, s}=a \varrho_{k}+A(s)=a \mu_{k}^{-1}+A(s)+a$. Здесь $\mu_{k}$ - собственное значение оператора $\widetilde{L}$, соответствующее его собственной функции $w_{k}=w_{k}(t), k=1,2,3, \ldots$ Пусть $v \in C[0, b]$ и равенство $\left(v, v_{k} ; H_{t}\right)=0$ справедливо при любом $k=1,2,3, \ldots$ Тогда при любом $k=1,2,3, \ldots$ справедливо равенство $\left(B v, w_{k} ; H_{t}\right)=0$. Откуда $B v=0$. Но оператор $B$ имеет обратный $B^{-1}$. Следовательно, $v=0$. Так как множество $C[0 \circ, b]$ плотно в $H_{t}$, в силу критерия $[3$, c. 16$]$ получим полноту в $H_{t} \mathrm{CC} \Phi$ оператора $L_{s}$. Учитывая структуру $\Phi$ СР уравнения (3), лемму 6 и представление СС $\Phi$ оператора $L_{s}$, заключаем, что набор $\left\{v_{k}, v_{k}^{*} ; k=1,2,3, \ldots\right\}$, где $v_{k}^{*}=t^{-\alpha} \overline{v_{k}(t)}$, образует биортонормированную систему в $H_{t}$. Так как $\left(v_{k}^{*} \in H_{t}\right) \Longleftrightarrow(\alpha<3 / 2)$, то условие минимальности в $H_{t}$ СС $\Phi$ оператора $L_{s}$ установлено [12, с. 306]. Завершая доказательство, осталось, с одной стороны, заметить, что для ССФ оператора $L_{s}$ справедливо представление (20), а операторная функция $B=B(\alpha)$ в зависимости от значений аргумента $\alpha$ обладает следуюшими ниже свойствами: $\left(0 \in \rho B^{-1} \cap C \sigma B\right) \Longleftrightarrow(\alpha \geqslant 0),\left(0 \in \rho B \cap C \sigma B^{-1}\right) \Longleftrightarrow(\alpha \leqslant 0)$, a с другой, воспользоваться соответствующими критериями, при выполнении которых $B$-система $[12$, с. 431$]$ является бесселевой или гильбертовой. 


\section{3. Общий случай}

3.1. Операторные уравнения и модельные операторы. Приступим к изучению операторного или, как еще говорят, дифференциально-операторного уравнения (1). Пусть $A$ - модельньй оператор, действующий в $\mathfrak{H}$, т.е. линейный замкнутый, вообще говоря, неограниченньй оператор с плотной в $\mathfrak{H}$ областью определения, полная система $\left\{\varphi_{s} ; s \in S\right\}$ собственных элементов $\varphi_{s}$ которого образует базис Рисса в $\mathfrak{H}[11$, c. 99]. Пусть также $H=H_{t} \otimes \mathfrak{H} ; \mathfrak{D}$ - линейное многообразие, состоящее из функций $u: \mathbb{R} \rightarrow \mathfrak{H}$ вида $u(t)=\sum_{s} v_{s}(t) \varphi_{s}$, где $v_{s}(t)$ из $\mathfrak{D}_{t}$ и суммирование проводится для конечного набора индексов $s \in S$. (Функция $u(t)$ (так же, как и операция $L\left(D_{t}, A\right)$ ) в формализме тензорных произведений $\left[3\right.$, с. 102] требует записи вида $u(t)=\sum_{s} v_{s}(t) \otimes \varphi_{s}$ $\left(L\left(D_{t}, A\right)=a t^{\alpha} D_{t}^{2} \otimes I+I \otimes A\right)$, которой, ради простоты, мы не следуем.)

ОПрЕДЕЛЕНИЕ 2. Элемент $u \in H$ называется обобщенным решением уравнения (1), где $f \in H$, если существует последовательность $\left\{u_{n}\right\}$ функций $u_{n}=u_{n}(t)$ из $\mathfrak{D}$ такая, что вьполнены равенства

$$
\lim _{n \rightarrow \infty}\left|u_{n}-u ; H\right|=\lim _{n \rightarrow \infty}\left|L\left(D_{t}, A\right) u_{n}-\lambda u-f ; H\right|=0 .
$$

Изучим функциональную зависимость спектра и свойств ССФ оператора $L: H \rightarrow H$, $L=L(\alpha, a, A)$, порожденного введенньг определением, от “параметров" $\alpha, a, A$. Обозначим символом $\widehat{L}_{s}$ сужение ("проекцию") оператора $L$ на подпространство

$$
H_{s}=H_{t} \otimes \varphi_{s}
$$

пространства $H$. Отметим, что $H_{s}$ - инвариантное подпространство оператора $\widehat{L}_{s}$.

Поскольку основным объектом, интересующим нас, является граничная задача, в дальнейшем роль $\mathfrak{H}$ будет играть пространство $H_{x}=H\left(V_{x}\right)$, где $V_{x}$ - ограниченная область пространства переменных $x \in \mathbb{R}_{x}^{n} \equiv \mathbb{R}^{n}$. Более того, при переходе к изучению операторного уравнения (1) удобно считать, что $A$ принадлежит специальному классу модельных операторов, а именно, $A$ является $\Pi$-оператором. Опишем интересующий нас класс операторов.

3.2. $\Pi$-операторы. Обозначим символом $V_{x} n$-мерный куб с ребром $2 \pi$ из пространства $\mathbb{R}_{x}^{n}, H_{x}=H\left(V_{x}\right)$ - гильбертово пространство комплекснозначных функций $w: \mathbb{R}_{x}^{n} \rightarrow \mathbb{C}$ с интегрируемым квадратом над $V_{x} ; \not{P} \infty$ - линейное многообразие гладких $\left(\mathscr{P}^{\infty} \subset C^{\infty}\left(\overline{V_{x}}\right)\right)$ периодических по всем переменным $\left(x_{1}, \ldots, x_{n}\right) \in \bar{V}_{x}$ комплекснозначных функций, плотное в $H_{x} ; S=\left\{s ; s \in \mathbb{Z}^{n}\right\}$. Полиному $A(s)$ с постоянными комплексными коэффициентами

$$
\begin{gathered}
A(s)=\sum_{|\alpha| \leqslant m} a_{\alpha} s^{\alpha} \\
a_{\alpha}=a_{\alpha_{1} \cdots \alpha_{n}} \in \mathbb{C}, \quad s^{\alpha}=s_{1}^{\alpha_{1}} \cdots s_{n}^{\alpha_{n}}, \quad \alpha_{k}=0,1,2, \ldots, \quad|\alpha|=\alpha_{1}+\cdots+\alpha_{n},
\end{gathered}
$$

поставим в соответствие дифференциальную операцию $A\left(-i D_{x}\right)$ таким образом, что будет вьполняться равенство

$$
A\left(-i D_{x}\right) \exp (i s \cdot x)=A(s) \exp (i s \cdot x), \quad s \cdot x=s_{1} x_{1}+\cdots+s_{n} x_{n} .
$$


Под оператором $A: H_{x} \rightarrow H_{x}$ понимается замыкание в $H_{x}$ первоначально заданной на функциях из $\mathscr{P} \infty$ операции $A\left(-i D_{x}\right)$. Совокупность экспонент $\{\exp (i s \cdot x), s \in S\}$ образует, очевидно, ортогональный базис и является одновременно набором собственных элементов оператора $A$, соответствующих набору собственных чисел $A(S)=\{A(s) ; s \in S\}$.

УТВЕРЖДЕНИЕ 1 [3]. Спектр $\sigma A$ оператора $A$ совпадает $c \overline{A(S)}$ - замыканием на $\mathbb{C}$ множества $A(S)$, образующего точечный спектр Р $\sigma A$ оператора $A$. При этом непрерывныц С $С A$ совпадает с разностью $\overline{A(S)} \backslash A(S)$.

3.3. Операционное исчисление для оператора $L$. Введенное пространство $H_{x}$ будет играть роль пространства $\mathfrak{H}$. Пространство $H=H_{t} \otimes H_{x}$ можно рассматривать как гильбертово пространство комплекснозначных функций $u: \mathbb{R}_{t} \times \mathbb{R}_{x}^{n} \rightarrow \mathbb{C}$ с интегрируемым квадратом над $V=V_{t} \times V_{x}$. Оператор $L$ можно рассматривать как замыкание в $H$ на множестве функций $u=u(t, x)$ из $\mathfrak{D}$, где $\varphi_{s}=\varphi_{s}(x)=\exp (i s \cdot x)$, операции $a t^{\alpha} D_{t}^{2}+A\left(-i D_{x}\right)$, т.е. $u$ принадлежит области определения $\mathfrak{D}(L)$ (замкнутого) оператора $L: H \rightarrow H$ и $L u=f$, если существует последовательность $\left\{u_{k}\right\}$ функций $u_{k}=u_{k}(t, x)$ из $\mathfrak{D}$ такая, что

$$
u_{k} \stackrel{H}{\rightarrow} u, \quad a t^{\alpha} D_{t}^{2} u_{k}+A\left(-i D_{x}\right) u_{k} \stackrel{H}{\rightarrow} f \quad \text { при } k \rightarrow \infty .
$$

Пусть $u \in \mathfrak{D}$ и $u=v(t) \cdot \varphi_{s}(x)$, т.е. $u \in H_{s} \cap \mathfrak{D}$. Тогда имеем

$$
L u=\widehat{L}_{s} u=\varphi_{s} \cdot L_{s} v, \quad\left|\widehat{L}_{s} u ; H\right|=\left|L_{s} v ; H_{t}\right| \cdot\left|\varphi_{s} ; H_{x}\right|
$$

где $v=v(t)$ и нормы берутся в пространствах $H, H_{t}$ и $H_{x}$ соответственно. Следовательно, операторы $\widehat{L}_{s}$ в рамках наших исследований можно заменить операторами $L_{s}$ и наоборот. Этим мы воспользуемся при изучении спектральных свойств оператора $L$. A именно, справедлива следуюшая теорема.

Теорема 1. Резольвентные множества операторов $L u L_{s}, s \in S$, связаны между собой следуюшим образом:

$$
(\lambda \in \rho L) \Longleftrightarrow\left(\lambda \in \bigcap_{s} \rho L_{s}\right) \wedge\left(\sup _{s}\left\|\left(L_{s}-\lambda\right)^{-1}\right\|<+\infty\right) .
$$

ДокаЗАтЕльство. Необходимость вытекает из определения операторов $\widehat{L}_{s}: \widehat{L}_{s} \subset L$, $s \in S$, и установленной взаимозаменяемости операторов $\widehat{L}_{s}$ и $L_{s}$.

Достаточность. Для произвольного элемента $f \in H$ его представление в виде ряда $f=\sum_{s} \widehat{f}_{s}, \widehat{f}_{s} \in H_{s}$, единственно. Из неравенства

$$
|u ; H|^{2}=\sum_{s}\left|\left(\widehat{L}_{s}-\lambda\right)^{-1} \widehat{f}_{s} ; H\right|^{2} \leqslant \sup _{s}\left\|\left(L_{s}-\lambda\right)^{-1}\right\|^{2} \cdot|f ; H|^{2}
$$

следует, что элемент $u=\sum_{s}\left(\widehat{L}_{s}-\lambda\right)^{-1} \widehat{f}_{s}$ является единственным решением уравнения $L u=\lambda u+f$.

Из доказанного немедленно вытекает 
СлЕДСТВИЕ 1. Если $\lambda \in \rho L$, то решение и уравнения (1) может быть представлено в виде ряда

$$
u=\sum_{s}\left(\widehat{L}_{s}-\lambda\right)^{-1} \widehat{f}_{s}=\sum_{s}\left(L_{s}-\lambda\right)^{-1} f_{s} \cdot \varphi_{s}
$$

əде $\widehat{f}_{s}-$ проекиия әлемента $f$ в $H_{s}: \widehat{f}_{s}=f_{s} \cdot \varphi_{s}, f_{s} \in H_{t} ; f_{s}=\left(f, \varphi_{s}^{*} ; H_{x}\right)$, $\left\{\varphi_{s}, \varphi_{s}^{*} ; s \in S\right\}-$ биортогональная в $H_{x}$ система функиий.

СлЕДСТвИЕ 2. Справедливы следующие высказывания:

$$
\begin{gathered}
(\lambda \in P \sigma L) \Longleftrightarrow\left(\exists s \in S \quad\left(\lambda \in P \sigma L_{s}\right)\right), \\
(\lambda \in C \sigma L) \Longleftrightarrow\left[\begin{array}{ll}
(\forall s \in S & \left.\left(\lambda \in \rho L_{s}\right)\right) \wedge\left(\sup _{s}\left\|\left(L_{s}-\lambda\right)^{-1}\right\|=+\infty\right), \\
(\forall s \in S & \left.\left(\lambda \notin P \sigma L_{s}\right)\right) \wedge\left(\exists s \in S \quad\left(\lambda \in C \sigma L_{s}\right)\right) .
\end{array}\right.
\end{gathered}
$$

ДоказАТЕЛЬСтво. Необходимость. Если $\lambda \in P \sigma L$, то из единственности разложения $u=\sum_{s} \widehat{u}_{s}, \widehat{u}_{s} \in H_{s}$, справедливого для любого $u \in H$, вытекает, что $\exists s \in S$ $\left(\lambda \in P \sigma \widehat{L}_{s}\right)$. В силу сделанного ранее замечания $\lambda \in P \sigma L_{s}$. Если $\lambda \in C \sigma L$, то оператор $\left(\widehat{L}_{s}-\lambda\right)^{-1}$ существует при любом $s \in S$. Откуда

$$
\left(\forall s \in S \quad\left(\lambda \in \rho L_{s}\right)\right) \vee\left(\forall s \in S \quad\left(\lambda \notin P \sigma L_{s}\right)\right) \wedge\left(\exists s \in S \quad\left(\lambda \in C \sigma L_{s}\right)\right)
$$

Eсли $\sup _{s}\left\|\left(L_{s}-\lambda\right)^{-1}\right\|<+\infty$, то из теоремы 1 имеем $\lambda \notin C \sigma L$.

Достаточность немедленно вытекает из определения операторов $\widehat{L}_{s}: \widehat{L}_{s} \subset L$, и установленной взаимозаменяемости операторов $\widehat{L}_{s}$ и $L_{s}$.

3.4. Спектр оператора $L$. Рассмотрения п 2.2 и 2.3 позволяют немедленно получить соответствующие результаты и в общем случае. А именно, справедливы следующие утверждения.

УТВЕРЖДЕНИЕ 2. Точечный спектр PбL оператора L может быть представлен формулой $\operatorname{P\sigma L}=\operatorname{P\sigma }\left(a t^{\alpha} D_{t}\right)+P \sigma A$.

При $\alpha<2$ совокупность собственных значений оператора $L$, определяемых уравненеием (7), является счетным (двухпараметрическим) множеством, допускающим при $k \rightarrow \infty$ асимптотическое представление вида

$$
\lambda_{k, s}=-a\left(\frac{(2-\alpha)^{2} \pi^{2}}{4 b^{2-\alpha}} k^{2}+\frac{\alpha(2-\alpha) \pi^{2}}{8 b^{2-\alpha}} k+\frac{\alpha(4-\alpha)}{16 b^{2-\alpha}}+\frac{\alpha^{2} \pi^{2}}{64 b^{2-\alpha}}\right)+A(s)+O\left(\frac{1}{k}\right)
$$

равномерное относительно параметра $s \in S$.

При $\alpha=2$ совокупность собственных значений оператора $L$ является двухпараметрическим множсеством мощности континуума: $\operatorname{P\sigma } L=\left\{\lambda_{\varrho, s}: \varrho \in \mathbb{P}, s \in S\right\}$, каждый әлемент которого допускает представление вида $\lambda_{\varrho, s}=a \varrho+A(s)$, где $\varrho \in \mathbb{P}, s \in S$. 


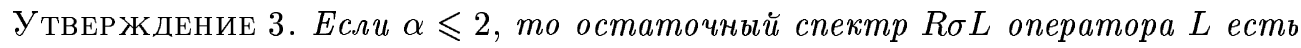
пустое мнохсество, т.е. $(\alpha \leqslant 2) \Longrightarrow(R \sigma L=\varnothing)$.

Приведем теперь пример свойства уравнения (1), отмеченного в п. 2.2 , для двумерного случая. Рассмотрим задачу Дирихле для уравнения

$$
t^{2} \frac{\partial^{2} u}{\partial t^{2}}+\frac{\partial^{2} u}{\partial x^{2}}=\lambda u+f(t, x)
$$

Обозначим через $T_{0}$ совокупность функций $u: \mathbb{R}^{2} \rightarrow \mathbb{C}$ класса $C^{0}(\bar{\Delta}) \cap C^{2}(\Delta)$, где $\Delta=V_{t} \times(0, \pi)$, удовлетворяющих условию Дирихле: $\left.u\right|_{\partial \Delta}=0$. Справедливо следующее

ПРЕДЛОЖЕНИЕ 6. Для любого $\lambda \notin \mathbb{P}_{0}^{\prime}=\mathbb{P}_{0}-1$ уравнение $(21)$ nри $f(t, x)=0$ не имеет нетривиального решения в классе $T_{0}$.

Если обозначить символом $\widetilde{K}_{D}$ оператор, порожденньй дифференциальным выражением, стоящим в левой части уравнения (21), областью определения которого является $T_{0}$, т.е. $\mathfrak{D}\left(\widetilde{K}_{D}\right)=T_{0}$ и

$$
\widetilde{K}_{D} u=t^{2} \frac{\partial^{2} u}{\partial t^{2}}+\frac{\partial^{2} u}{\partial x^{2}},
$$

а $K_{D}$ - его замыкьние в $\mathscr{L}_{2}(\Delta)$, то предложение 6 может быть записано и в следующей форме: $P \sigma \widetilde{K}_{D}=\mathbb{P}_{0}^{\prime}$, причем $P \sigma \widetilde{K}_{D} \subset P \sigma K_{D}$, где “С" - знак собственного подмножества.

Отметим, что существование решения задачи Дирихле для уравнения эллиптического типа

$$
t^{\alpha} \frac{\partial^{2} u}{\partial t^{2}}+\frac{\partial^{2} u}{\partial x^{2}}+a \frac{\partial u}{\partial t}+b \frac{\partial u}{\partial x}+c u=0
$$

с аналитическими коэффициентами $a, b, c(c \leqslant 0)$, вырождающегося на гранище рассматриваемой области $\Delta$, было изучено в [13].

3.5. Свойства ССФ оператора $L$. При изучении свойств ССФ оператора $L$ ограничимся, как и для обыкновенного дифференциального оператора, случаем $\alpha<2$.

УТВЕРЖДЕНИЕ 4. Пусть $\alpha<2$.

1. Собственная функиия $u_{k, s}(t, x)$ оператора $L$, соответствующая собственному значению $\lambda_{k, s}=a \varrho_{k}+A(s)$, имеет вид $u_{k, s}(t, x)=v_{1}\left(t, \varrho_{k}\right) \cdot \varphi_{s}(x)$, әде $v_{1}\left(t, \varrho_{k}\right)$ мохсет быть представлена либо в виде степенного ряда (см. (3а)), либо в виде контурного интеграла (см. (8а)).

2. Совокупность $\left\{u_{k, s} ; k \in \mathbb{N}, s \in S\right\}$ собственных функиий $u_{k, s}=u_{k, s}(t, x)$ оператора $L=L(\alpha, a, A)$ образует:

а) полную систему в $H$;

б) (минимальную систему в $H) \Longleftrightarrow(\alpha<3 / 2)$;

в) $($ гильбертову систему в $H) \Longleftrightarrow(0 \leqslant \alpha<3 / 2)$;

г) (бесселеву систему в $H) \Longleftrightarrow(\alpha \leqslant 0)$. 
ДокАЗАТЕЛЬство. Представление собственных функций оператора $L$ очевидно. Доказательство полноты в $H$ CС $\Phi$ оператора $L$, основанное на полноте в $H_{t}$ CС $\Phi$ оператора $t^{\alpha} D_{t}$, проводится так же, как и в [4]. Оставшиеся свойства СС $\Phi$ оператора $L$, вытекающие из аналогичных свойств СС $\Phi$ оператора $L$ в одномерном случае, доказываются тем же методом, каким оно было проведено для дифференциально-операторного уравнения первого порядка по $t$ в работе [14].

Отметим, что проведенные рассуждения нетрудно перенести на тот случай, когда $a$, так же как и $A$, является М-оператором, т.е. $a, A: \mathfrak{H} \rightarrow \mathfrak{H}$, причем, естественно, они должны обладать общей полной СС $\Phi$, образующей базис Рисса в $\mathfrak{H}$.

В заключение автор выражает глубокую благодарность профессору А. А. Дезину и профессору В. К. Романко за внимание к работе.

\section{СПИСОК ЦИТИРОВАННОЙ ЛИТЕРАТУРЫ}

[1] Соболев С. Л. Некоторые приложения функционального анализа в математической физике. Л.: Изд-во ЛГУ, 1955.

[2] Хилле Э., Филлипс Р. Функциональный анализ и полугруппы. М.: ИЛ, 1962.

[3] Дезин А. А. Общие вопросы теории граничных задач. М.: Наука, 1980.

[4] Дезин А. А. О слабой и сильной иррегулярности // Дифференц. уравнения. 1981. Т. 17. № 10. C. 1851-1858.

[5] Романко В. К. О собственных значениях краевых задач для некоторых уравнений, меняющих тип // Дифференц. уравнения. 1983. Т. 19. №10. С. 1759-1764.

[6] Kornienko V. V. // International Conference on Some Topics of Mathematics. Abstracts. Samarkand, 1996. P. 84.

[7] Дезин А. А. Вырождающиеся операторные уравнения // Матем. сб. 1981. Т. 115 (157). № $3(7)$. C. $323-336$.

[8] Тепоян Л. П. Вырождающиеся дифференциально-операторные уравнения четвертого порядка. Дисс. ... к.ф.--м.н. М., 1986.

[9] Степанов В.В. Курс дифференциальных уравнений. М.: Гостехиздат, 1945.

[10] Смирнов В. И. Курс высшей математики. Т. 3. Ч. 2. М.: Наука, 1974.

[11] Наймарк М. А. Лиейные дифференциальные операторы. М.: Наука, 1969.

[12] Качмаж С., Штейнгауз Г. Теория ортогональных рядов. М.: ГИФМЛ, 1958.

[13] Келдыш М. В. О некоторых случаях вырождения уравнений эллиптического типа на границе области // Докл. АН СССР. 1951. Т. 77. № 2. С. 181-183.

[14] Корниенко В.В.К слабой и сильной иррегулярности // Сиб. матем. ж. 1996. Т. 37. №3. C. 599-609.

Самаркандский государственный университет им. А. Навои

Поступило

E-mail: korni@mchmat.samuni.silk.org

06.03.1997

Исправленный вариант

30.11.1999 